\title{
Prognostic and diagnostic value of EEG signal coupling measures in coma
}

Frederic Zubler ${ }^{*}$, Christa Koenig ${ }^{1}$, Andreas Steimer ${ }^{1}$, Stephan M. Jakob ${ }^{2}$, Kaspar A. Schindler ${ }^{1}$, Heidemarie Gast ${ }^{1}$

${ }^{1}$ Department of Neurology, Bern University Hospital, University of Bern, Bern, Switzerland

${ }^{2}$ Department of Intensive Care Medicine, Bern University Hospital, University of Bern, Bern, Switzerland

\section{* Corresponding author:}

Frederic Zubler

Department of Neurology

Bern University Hospital - Inselspital

Freiburgstrasse 4

3010 Bern

Switzerland

Tel.: +4178647 7162

E-mail: frederic.zubler@gmail.com 


\begin{abstract}
Objective: Our aim was to assess the diagnostic and predictive value of several quantitative EEG (qEEG) analysis methods in comatose patients.

Methods: In 79 patients, coupling between EEG signals on the left-right (interhemispheric) axis and on the anterior-posterior (intra-hemispheric) axis was measured with four synchronization measures: relative delta power asymmetry, crosscorrelation, symbolic mutual information and transfer entropy directionality. Results were compared with etiology of coma and clinical outcome. Using cross-validation, the predictive value of measure combinations was assessed with a Bayes classifier with mixture of Gaussians.

Results: Five of eight measures showed a statistically significant difference between patients grouped according to outcome; one measure revealed differences in patients grouped according to the etiology. Interestingly, a high level of synchrony between the left and right hemisphere was associated with survival, whereas higher synchrony between anterior and posterior brain regions was associated with mortality on intensive care unit. The combination with the best predictive value reached an AreaUnder the Curve of 0.875 (for patients with post anoxic encephalopathy: 0.946). Conclusion: EEG synchronization measures can contribute to clinical assessment, and provide new approaches for understanding the pathophysiology of coma. Significance: Prognostication in coma remains a challenging task. qEEG could improve current multi-modal approaches.
\end{abstract}

\title{
Keywords
}

Coma, prognostication, quantitative EEG, synchronization, Bayes classifier. 


\section{Introduction}

Assessment of comatose patients is a notoriously difficult, but essential task. After initial stabilisation of vital functions, identifying the etiology of coma is necessary so that an appropriate therapy can be initiated (Edlow et al., 2014). In absence of rapid recovery of consciousness, it is of highest importance to evaluate the prognosis of comatose patients. Identifying those patients with no hope of recovery would allow considering withdrawal of support, both for ethical and economical reasons (Young, 2009; Howard et al., 2011).

Currently, no single method is capable of adequate etiological diagnosis and prognostication. Therefore, multimodal algorithms combining clinical examination, electroencephalography (EEG), evoked potential, and biomarkers have been proposed, aiming at improving prognostic indicators in coma (Bassetti et al., 1996; Oddo and Rossetti, 2014; Rossetti et al, 2010; Wijdicks et al., 2006). The majority of these studies has focused on patients with hypoxic-ischemic brain injury after cardiac arrest; the reasons being high incidence and still a very high proportion of nonsurvivors (Go et al., 2013). While therapeutic hypothermia (TH) has concurred to improve the prognosis for these patients, it has also further complicated the prognostication (Crepeau et al., 2013, Kamps et al., 2013; Rossetti et al., 2010). Multimodal prediction tools keep improving, even during TH (Oddo and Rossetti, 2014; Tjepkema-Cloostermans et al., 2015). However, the quest for an optimal algorithm continues, and novel complementary approaches are called upon. Quantitative EEG (qEEG) methods are emerging as possible candidates.

In essence, qEEG methods consist of computer-based analysis of EEG signals. Many of these methods are based on spectral decomposition of EEG signals, some of which have been applied successfully to automatic seizure detection (Gotman et al., 1997; van Putten et al., 2005), to detection of arterial spasm after subarachnoid hemorrhage (Forman and Claassen, 2013), or as a prognostication tool after stroke (van Putten, 2007; van Putten and Tavy, 2004). Other qEEG methods have also been proposed as "surrogate encephalographer", allowing non-specialists to recognize conventional EEG patterns as a neurologist would (Cloostermans et al., 2011). Several qEEG 
methods have helped to refine the recognition of classical patterns, identifying for instance subtypes of status epilepticus (Rundgren et al., 2010) or burst suppression patterns (Hofmeijer et al., 2014). Finally, several methods such as amplitudeintegrated EEG (Rundgren et al., 2010) or the recently proposed cerebral recovery index (Tjepkema-Cloostermans et al., 2013) have been applied to predict outcome of patients after cardiac arrest by identifying global (for the former) or more local (for the latter) features of the EEG signal similar to the ones that a trained electroencephalographer would focus on.

By contrast, we wanted to investigate the utility of qEEG methods for extracting EEG features that are not easily accessible to a human observer. The motivation to do so was to provide the treating physician with additional information about a patient's condition, which could be for instance included into future multimodal prognostic algorithms. Therefore, beside an index based on spectral decomposition, we focused here on another class of qEEG methods, namely signal coupling measures. These measures detect synchronization between EEG signals, and have thus been used so far mainly to define so-called "functional brain networks" (Kramer, 2010; Stam and van Straaten, 2012; van Diessen et al., 2013). Since EEG signals do not always have a dominant frequency, synchronization is not meant in the classical sense of convergence of phase and frequency, but in the more general sense of symmetrical or asymmetrical interdependence between signals (Rulkov et al., 1995; Stam et al., 2002). Such methods are capable of distinguishing between conscious state, minimally conscious state and vegetative state (King et al., 2013; Sitt et al., 2014). In this retrospective study we show that qEEG coupling measures can contribute to the assessment of comatose patients, and that beside their diagnostic and prognostic value, these methods might contribute to a better understanding of the pathophysiology of coma.

\section{Methods}

\subsection{Patients and study design}

This retrospective study was conducted in the 30-bed intensive care unit and the 15bed intermediate care unit (ICU) of the University Hospital of Bern, in Switzerland. 
Consecutive comatose patients (aged $>16$ years) who had had an EEG for medical reasons between January 2008 and January 2012 were included. Coma was defined based on the different elements of the Glasgow Coma Scale (GCS), namely: eye response value $=1$, verbal response value $<=2$, and motor response value $<=4$. Only patients with good quality EEG recordings were included. We excluded patients suffering of brain hemorrhages or traumatic head injury, or with recent history of brain surgery. In case of multiple EEGs, only the first was analyzed. In patients who had been treated with therapeutic hypothermia, EEGs were performed after rewarming under normothermic condition. This study was approved by the cantonal ethics committee of Canton of Berne.

The etiology for coma was categorized into hypoxic/anoxic, and non-hypoxic. In the hypoxic/anoxic group were patients with cardiac arrest, hemorrhagic shock, large ischemic brain lesions or other anoxic conditions (e.g. suffocation). The non-hypoxic group was subdivided into the etiologies infectious, metabolic or drug-related, and epileptic. Concerning the clinical outcome, patients were grouped into alive or deceased at discharge from the ICU. Patients in the group "alive" were heterogeneous and included patients who had fully recovered as well as patients with impaired consciousness. The group of deceased patients consisted of patients for whom the decision to withdraw medical support and life sustaining therapies was made (those patients received palliative treatment) and patients who died instead of treatment. The latter group consisted only of two patients, thus the group of deceased patients was not split. The decision to withdraw life support was made by the treating physicians and the patients' surrogates if available. The most relevant determinants of this decision were a very low probability of survival, a high probability of severely impaired cognitive function. The quantitative EEG analysis was not available at the time of the decision to continue or withdraw treatment.

\subsection{Data collection}

Information about GCS score and medication (including sedation) at recording time was taken from the EEG report that was signed by the attending neurologist. Additional clinical and demographical data used for this work were collected in the electronic patient documentation system of the Bern University Hospital. For EEG 
recordings 21 electrodes were used (19 active electrodes placed on the scalp according to the international 10-20 system, one reference electrode, one ground electrode). Each recording was performed for a length of approximately 20 minutes, of which the first segments of 5 minutes without obvious artefacts were analyzed. We used a NicoletOne recording system with a C64 amplifier (VIASYS Healthcare, Inc., Madison, WI, U.S.A.). The sample rate was $500 \mathrm{~Hz}$.

\subsection{Quantitative EEG analysis.}

We considered four different bipolar derivations, corresponding to four different brain regions, namely F3-P3 for the left hemisphere, F4-P4 for the right hemisphere, F3-F4 for the anterior region, and P3-P4 for the posterior region (Fig. 1). Four different bivariate quantitative EEG (qEEG) methods were used to compute signal-coupling value between the left and right bipolar derivations, and between the anterior and posterior bipolar derivation. The four qEEG methods applied were (1) relative delta power asymmetry, (2) cross-correlation, (3) symbolic mutual information and (4) symbolic transfer entropy directionality. Each qEEG synchronization measure was repeatedly computed for epochs consisting of non-overlapping time windows of 10 seconds (= 5000 sampling points); the average value over 30 epochs was used in statistical analysis. Signal analysis was done with MATLAB Version R2012a (MathWorks). Except for computation of relative delta power, the EEG signals were digitally band-passed filtered between 0.5 and $20 \mathrm{~Hz}$ before quantitative analysis, in order to remove DC shifts and high frequency artefacts.

\subsubsection{Relative delta power asymmetry (RDP)}

While power spectrum analysis is primarily a univariate measure, it can be used to define bivariate asymmetry indices. The first qEEG measure we used was the asymmetry in relative delta power (RDP). This particular frequency band was chosen because global delta power is associated with loss of consciousness in sleep, anesthesia, and coma of various causes (Kaplan and Bauer, 2011). Focal delta slowing is also the hallmark of several severe neurological conditions such as ischemic stroke (Jordan 2004), and a focal increase in delta power has been used for instance for EEG monitoring after subarachnoid hemorrhage (Claassen et al., 2004). We defined relative delta power as the ratio of power in the delta band $(0.5-4 \mathrm{~Hz})$ to the total 
power in the frequency interval $0.5-20 \mathrm{~Hz}$. Power was computed over each 10 seconds epoch at a resolution of $0.5 \mathrm{~Hz}$ with the pwelch method from MATLAB, which uses the Goertzel algorithm over eight Hanning windows (50\% overlap). RDP was first computed on the four bipolar derivations as noted above, and used to define two asymmetry indices, namely the left-right RDP asymmetry-index: $\mathrm{RDP}_{\mathrm{LR}}=$ $\left|\left(\mathrm{RDP}_{\mathrm{L}}-\mathrm{RDP}_{\mathrm{R}}\right) /\left(\mathrm{RDP}_{\mathrm{L}}+\mathrm{RDP}_{\mathrm{R}}\right)\right|$, and the anterior-posterior RDP asymmetry index: $\mathrm{RDP}_{\mathrm{AP}}=\left|\left(\mathrm{RDP}_{\mathrm{A}}-\mathrm{RDP}_{\mathrm{P}}\right) /\left(\mathrm{RDP}_{\mathrm{A}}+\mathrm{RDP}_{\mathrm{P}}\right)\right|$. The vertical bars indicate that we used the absolute value, so that only the magnitude of the asymmetry index, and not the location of maximum or minimum value was taken into account. The indices took a value between 0 , if power in the delta band was equal in both EEG signals (left and right, or anterior and posterior), and +1 , if frequencies in the delta band were found only at one end of the left-right or anterior-posterior axis.

\subsubsection{Cross-correlation (CC)}

As second measure we used zero-lag cross-correlation (CC), a bivariate measure, which has been extensively used to study EEG signals pairwise (Kramer et al., 2010) and global synchronization during epileptic seizures (Schindler et al., 2007). Intuitively, two signals have a high cross-correlation if they are scaled versions of one another. $\mathrm{CC}$ was computed between the bipolar derivations representing left and right hemisphere $\left(\mathrm{CC}_{\mathrm{LR}}\right)$, as well as between the bipolar derivations representing the anterior and posterior brain regions $\left(\mathrm{CC}_{\mathrm{AP}}\right)$. Considering that anticorrelation is also an indication of synchrony, we considered only the absolute value of CC. Boundaries for $\mathrm{CC}$ were thus 0 , in case of total absence of correlation between signals, and +1 , if the two signals were perfect scaled copies of each other.

\subsubsection{Symbolic transformation.}

Whereas RDP and CC values were directly computed on the bipolar EEG signals (repeated measures of voltage expressed in $\mathrm{mV}$ ), the following qEEG measures were applied to a symbolized version of the signals (Daw et al., 2003). That is, the bandpass filtered EEG was transformed into a chain of symbols, reflecting the temporal dynamics of the signal. The advantages of this procedures are, first, that the symbolization offers a natural binning for statistical estimation needed by the two subsequently described qEEG methods, and second, the extreme resistance to noise (Rummel et al., 2013). In this work we used a bit-string symbolization (see 
Supplementary Material for details). In short, a bit string is a sequence of binary values, where 1 represents an increase, and 0 a decrease in the amplitude of the original EEG signal. A symbol was then defined as a "word" of 4 bits, taken at an interval of 5 time steps, i.e. every $10 \mathrm{~ms}$ (that is, the symbol at time $t$ consists of bits from time $t, t-10 \mathrm{~ms}, t-20 \mathrm{~ms}$, and $t-30 \mathrm{~ms}$; for a visual representation see Figure 1 in Zubler et al., 2014).

\subsubsection{Symbolic mutual information (MI)}

Mutual information is a bivariate information theoretic measure, which quantifies the decrease of uncertainty about the current value of one signal caused by the knowledge of a second signal (Cover and Thomas, 2006). Unlike CC, MI also detects non-linear interactions. As already mentioned, we used the symbolic mutual information, namely the mutual information on the strings obtained by symbolic transformation. Values were normalized by the joint entropy between the two symbolic strings, so that the boundaries were 0 , if the two symbolized signals (and their corresponding EEG signals) were completely independent, and +1 , if the two symbolized EEG were so strongly interdependent that knowing the value of one signal also resulted in the knowledge of the other one. We computed symbolic mutual information between the left and right bipolar derivations $\left(\mathrm{MI}_{\mathrm{LR}}\right)$, as well as between the anterior and posterior bipolar derivations $\left(\mathrm{MI}_{\mathrm{AP}}\right)$. See Supplementary Material for detailed implementation.

\subsubsection{Symbolic transfer entropy directionality (TE)}

In short, TE is a measure of the information flow from one signal to another signal (Schreiber, 2000). Unlike the previous measures, TE is a directed measure. That is, TE from $\mathrm{x}$ to $\mathrm{y}$ is as a basic principle different from TE from $\mathrm{y}$ to $\mathrm{x}$. As for MI, we applied TE after symbolization (Staniek and Lehnertz, 2008); for details concerning the implementation we refer to Supplementary Material and to (Zubler et al., 2014). The information flow was computed from left to right, from right to left, from anterior to posterior, and from posterior to anterior brain regions. These results were used to define two asymmetry indices $\mathrm{TE}_{\mathrm{LR}}=\left|\left(\mathrm{TE}_{\mathrm{L} \text { to } \mathrm{R}}-\mathrm{TE}_{\mathrm{R} \text { to } \mathrm{L}}\right) /\left(\mathrm{TE}_{\mathrm{L} \text { to } \mathrm{R}}+\mathrm{TE}_{\mathrm{R} \text { to } \mathrm{L}}\right)\right|$, and $\mathrm{TE}_{\mathrm{AP}}=\left(\mathrm{TE}_{\mathrm{A} \text { to } \mathrm{P}}-\mathrm{TE}_{\mathrm{P} \text { to } \mathrm{A}}\right) /\left(\mathrm{TE}_{\mathrm{A} \text { to } \mathrm{P}}+\mathrm{TE}_{\mathrm{P} \text { to } \mathrm{A}}\right)$. Note that in the left-right axis we considered the absolute-valued asymmetry index, which took on values between 0 , in case of balanced information flow, and 1, in case where inter-hemispheric information flow was purely unidirectional. In the anterior-posterior axis, by contrast, we 
considered the signed-valued asymmetry index. The reason being the net frontal-toparietal information flow in awake human and animals subjects observed in previous studies using transfer entropy or symbolic transfer entropy (Imas et al., 2005; Lee et al., 2009; Lee et al., 2013). $\mathrm{TE}_{\mathrm{AP}}$ took on values between -1 (if information flow was only detected from posterior to anterior) and +1 (if the information flow was exclusively flowing in the opposite direction); values around 0 indicated a balanced information flow.

\subsection{Statistical Analysis}

Differences between groups of patients who survived and patients who deceased were assessed with a non-parametric Mann-Whitney $U$ test for difference of mean ranks. Statistical significance of p-values was confirmed with Benjamini-Krieger-Yekutieli false discovery rate control for multiple comparisons (for implementation in MATLAB see (Groppe et al., 2011)). Differences between the four etiologies were assessed with a non-parametric Kruskal-Wallis test; if the 5\% significance level was attained, Tukey-Kramer post-hoc multi-comparison tests were performed (MATLAB function multcompare).

\subsection{Bayes Classifier}

A binary Bayes classifier was applied to select the best combination of qEEG coupling measures for prognostication of clinical outcome, and to quantify its predictive value. In short, binary Bayes classifiers use part of the data (the "training set") to learn how to associate each data point with a probability for belonging to one of two groups. A so-called discrimination threshold is then chosen, which defines the actual boundary between the two groups. Based on this threshold the classifier can attribute new data points, which did not belong to the training set, to one of the two groups. We recall that the receiver operating characteristic (ROC)-curve is a plot of the false positive rate (FPR) and the false negative rate (FNR) as the discrimination threshold is moved. In our case, for each of the $2^{8}=256$ possible combinations of qEEG measures, we trained a Bayes classifier using a mixture of Gaussians to discriminate patients who survived from those patients who deceased on the ICU. We used only 2 Gaussians per class to reduce the number of parameters and avoid overfitting. For each measure combination, we used a leave-one-out cross-validation 
method. That is, the training was repeatedly conducted on all possible 79 data sets that consisted of all but one patient. Errors in predicting the outcome of the respective remaining single patient were used to compute the ROC curve for each classifier. By definition, FPR was the fraction of surviving patients in the group of patients classified as non-survivor, and FNR was the fraction of patients who deceased in the group of patients classified as survivor.

The general predictive value of each combination of each qEEG measure was first determined based on the area under the ROC curve (AUC). For practical use, however, ROC curves alone are not sufficient, and one has to define a specific threshold (or equivalently, a point on the ROC-curve) on which the classifier will operate. Often, the threshold chosen is the one that minimizes the so-called L1distance between the ROC-curve and the point FPR $=0$, TPR $=1$ in ROC space. This is equivalent to finding the point on the ROC-curve that minimizes the value $K=F P R$ + FNR. However, this might not be the optimal point, since FPR and FNR can have a different relative importance due to medical and economical reasons. In the context of comatose patients, it can be argued that falsely classifying a patient as having no chance to recover (false positive) is 'ethically more costly' than the reversed case, that is, failure to identify a patient who is likely to decease (false negative) or equivalently, that specificity is more important than sensitivity. We used a new parameter, lambda, to assign different subjective weights to FPR and FNR by defining $\mathrm{K}_{\text {lambda }}=$ FPR x lambda + (1-lambda) x FNR, and having the classifier operating with the threshold value that minimizes $\mathrm{K}_{\text {lambda }}$ on the ROC-curve. For each of the 256 feature combinations several values of lambda between 0 (FNR was more important) and 1 (FPR was more important) were used. These values for lambda were $1 / 6,1 / 3,1 / 2,2 / 3,5 / 6$. Besides, performances were measured at the lambda value that maximizes the accuracy (the fraction of patients who are correctly classified).

\section{Results}

\subsection{Patient demographics}


EEG recordings of 106 patients were examined. 27 patients were excluded because of insufficient recording quality. 79 patients, 35 females (45\%), with a good quality EEG recording were included (Supplementary Table S1). The mean age of included patients (+/- 1 standard deviation) was $58+/-16$ years (range 19-84 years). 39 of the included patients left the ICU alive and awake, 7 were alive but still comatose, stuporous, or with fluctuating consciousness, adding up to a total of 46 survivors (58\%). 33 patients died in the ICU (42\%), 31 after life support had been withdrawn, and 2 died instead of treatment. The underlying etiology of coma was hypoxic/anoxic in 38 and non-hypoxic in 41 patients. Of those with non-hypoxic etiology the cause of coma was primarily epileptic in 25 patients, infectious in 11, metabolic in three, and pharmacological in two patients (hereinafter, the categories metabolic and pharmacologic are grouped). The survival rate was significantly worse in case of hypoxic $(9 / 38=24 \%)$ compared to non-hypoxic origin of coma $(37 / 41=90 \%)$. By contrast, there was no significant age difference between survivors $(57+/$ - 16 years) and non-survivors $(59+/-15$ years; $\mathrm{p}=0.87)$.

EEGs were recorded from within the first hour after admission to the ICU to up to 23 days later (all but 5 EEGs were recorded within the first 4 days at the ICU; for the subgroup with post-hypoxic/anoxic origin of coma all but one patient had an EEG within 3 days after admission). The timing of the EEG recordings after admission to the ICU was not significantly different between patients who survived $(1.70+/-1.6$ days) and patients who did not survive $(1.76+/-1.0 ; \mathrm{p}=0.39$; for the 5 patients whose EEG was recorded more than 4 days after admission the value was set to 5).

Details concerning medication are listed in Supplementary Table S1. Patients were mainly sedated with propofol. Propofol was administrated during EEG recording in $26 \%$ of all patients (in $28 \%$ of survivors, and in $24 \%$ or non-survivors); it was discontinued at beginning of recording or in the minutes before in $37 \%$ of cases (in $37 \%$ of survivors, in $36 \%$ of non-survivors). The EEG was performed without propofol in the remaining 37\% (and in 35\% of surviving and in $39 \%$ of nonsurviving) patients. The average dose of propofol was not significantly different between survivors (average dose given during EEG: 48 +/- $96 \mathrm{mg} / \mathrm{h}$; average dose during or discontinued before EEG: 97 +/- $120 \mathrm{mg} / \mathrm{h}$ ) and non-survivors (only during EEG: 72 +/- 109; $\mathrm{p}=0.43$; during or before EEG: $138 \mathrm{mg} / \mathrm{h}+/-124 ; \mathrm{p}=0.16$ ). 


\subsection{Group analysis}

We used four quantitative methods to characterize the coupling between scalp EEG signals in the left-right (inter-hemispheric) axis, and in the anterior-posterior (intrahemispheric) axis in comatose patients. Fig. 2 shows four individual examples, with EEG signals displayed as for classical analysis, together with a vector of the eight qEEG measures (in this representation normalized by the highest value for each measure). Values of each coupling measure were compared between patients grouped according to the clinical outcome, that is, if the patient survived (S) or deceased (D) during their stay in the ICU, as well as according to the coma etiology: hypoxic /anoxic, infectious, metabolic/pharmacological/toxic, and epileptic (Fig. 3 and 4). Most of the literature on EEG prognostication in coma concerns post-hypoxic encephalopathy. We thus performed subgroup analysis for patients within this group, in order to investigate if our approach was valid when restricted to this etiology of coma. For subgroups with other aetiologies the number of patients and unbalanced outcome did not allow for subgroup analysis.

Values are expressed as mean + /- standard deviation. Uncorrected p-values are given; statistical significance after false-discovery rate (FDR) control is indicated with an asterisk $(*)$.

Relative delta power: In left-right axis, the absolute-valued relative delta power asymmetry showed no significant differences between the group of patients who survived, and the group of patients who deceased while on the ICU (RDP LR $_{\text {: }} \mathrm{S}=$ $0.016+/-0.017, \mathrm{D}=0.034+/-0.052, \mathrm{p}=0.51$; Fig. $3 \mathrm{~A}$ ). In the anterior-posterior axis, however, the asymmetry was significantly higher in the group of deceased patients $\left(\mathrm{RDP}_{\mathrm{AP}}: \mathrm{S}=0.027+/-0.037, \mathrm{D}=0.098+/-0.13, \mathrm{p}=0.00012 *\right.$; Fig. 3B $)$.

A similar trend was observed when we performed the analysis on the subgroup of 38 patients with an anoxic/hypoxic etiology of coma: $\mathrm{RDP}_{\mathrm{AP}}$ was on average higher in patients who deceased on the ICU, whereby the difference was no longer significant 
after FDR control $(S=0.03+/-0.037, D=0.01+/-0.013, p=0.014$; Supplementary Figure S1A,B).

No statistically significant differences in relative delta power asymmetry were found between patients grouped according to the etiology of coma. However, the difference was close to significant level for $\mathrm{RDP}_{\mathrm{AP}}$, patients with hypoxic/anoxic origin of coma showing on average a more pronounced asymmetry than patients with a primarily epileptic origin of coma $(H=0.082+/-0.12, E=0.023+/-0.032, p=0.053$, Fig. 4B $)$.

Cross-correlation: Both the inter-hemispheric and intra-hemispheric cross-correlation values were significantly different in patients grouped according to the clinical outcome. Interestingly to note, while a higher level of correlation between left- and right hemisphere was found in patients who deceased $\left(\mathrm{CC}_{\mathrm{LR}}: \mathrm{S}=0.71+/-0.096, \mathrm{D}=\right.$ $0.79+/-0.17, \mathrm{p}=0.0073^{*}$, Fig. 3C), a higher correlation between the anterior and the posterior brain regions was associated with survival $\left(\mathrm{CC}_{\mathrm{AP}}: \mathrm{S}=0.64+/-0.11, \mathrm{D}=\right.$ $0.60+/-0.15, p=0.020 *$; Fig. 3D). The group differences were no longer significant when considering only patients with post anoxic encephalopathy $(\mathrm{p}=0.22$ and $\mathrm{p}=$ 0.16, respectively; Supplementary Figure S1C,D).

$\mathrm{CC}_{\mathrm{LR}}$ was significantly higher in the group of patients with hypoxic/anoxic encephalopathy than in patients with a primarily epileptic or infectious etiology of coma $(\mathrm{H}=0.78+/-0.15, \mathrm{E}=0.66+/-0.11, \mathrm{I}=0.73+/-0.046, \mathrm{p}=0.00052$, Fig. 4C). By contrast, $\mathrm{CC}_{\mathrm{AP}}$ values were on average lower after hypoxic/anoxic brain lesion than in the primarily epileptic group; however this difference did not reach statistical significance $(\mathrm{H}=0.59+/-0.14, \mathrm{E}=0.66+/-0.11, \mathrm{p}=0.057$, Fig. 4D).

Symbolic mutual information: No statistically significant difference was found in inter- and intra-hemispheric mutual information between group of patients with different outcome $\left(\mathrm{MI}_{\mathrm{LR}}: \mathrm{S}=0.077+/-0.031, \mathrm{D}=0.11+/-0.077, \mathrm{p}=0.32\right.$, Fig. 3E; $\mathrm{MI}_{\mathrm{AP}}: \mathrm{S}=0.057+/-0.024, \mathrm{D}=0.065+/-0.033, \mathrm{p}=0.32$, Fig. $\left.3 \mathrm{~F}\right)$. Restricting the analysis to patients with hypoxic/anoxic encephalopathy did not show differences either between patients who survived and patients who deceased while on the ICU (Supplementary Figure S1E,F). 
Similarly, no difference in mutual information was found according to the etiology of coma $\left(\mathrm{MI}_{\mathrm{LR}}: \mathrm{p}=0.27\right.$, Fig. 4E; $\mathrm{MI}_{\mathrm{AP}}: \mathrm{p}=0.52$, Fig. 4F).

Symbolic transfer entropy: In left-right axis the absolute-valued asymmetry-index was significantly larger in patients who deceased while in the ICU than in survivor patients ( $\mathrm{TE}_{\mathrm{LR}}: \mathrm{S}=0.041+/-0.038, \mathrm{D}=0.084+/-0.091, \mathrm{p}=0.04 *$; Fig. 3G).

Conversely, in the anterior-posterior axis the signed asymmetry-index was significantly higher in patients who survived the acute phase, these patients showing a stronger net information flow from frontal to parietal brain regions $\left(\mathrm{TE}_{\mathrm{AP}}: \mathrm{S}=0.05+/-\right.$ $0.079, \mathrm{D}=0.0023+/-0.092, \mathrm{p}=0.015^{*}$, Fig. 3H). Similar trends were observed in the subgroup of hypoxic patients, whereby the results were no longer significant after FDR control (TE $\mathrm{TR}_{\mathrm{LR}} \mathrm{S}=0.026, \mathrm{D}=0.09, \mathrm{p}=0.011$ TE $_{\mathrm{AP}}: \mathrm{S}=0.051, \mathrm{D}=0.0078, \mathrm{p}=$ 0.025; Supplementary Figure S1G,H).

No significant difference was observed between groups of patients with different etiologies of coma (TE $\mathrm{TR}_{\mathrm{LR}} \mid \mathrm{p}=0.6$; Fig. 4G; TE $\mathrm{AP}: \mathrm{p}=0.15$; Fig. $\left.4 \mathrm{H}\right)$.

\subsection{Classifier for prognostication}

The prognostic value of each 256 possible combination of qEEG measures was tested with a Bayes classifier. The performances were first assessed based on the area under the ROC-curve (AUC). The highest AUC value was 0.875 , and was obtained by a combination consisting of six measures, namely $\mathrm{RDP}_{\mathrm{AP}}, \mathrm{CC}_{\mathrm{LR}}, \mathrm{CC}_{\mathrm{AP}}, \mathrm{MI}_{\mathrm{LR}}, \mathrm{MI}_{\mathrm{AP}}$, $\mathrm{TE}_{\mathrm{AP}}$ (Fig. 5A). In addition, we observed that several other combinations reached an almost equivalently score (between the combinations on $1^{\text {st }}$ and on $10^{\text {th }}$ rank, the difference in AUC was 2.3\%; Supplementary Figure S2A). Thus, before concluding that these six measures had the best prognostic value, and that $\mathrm{RDP}_{\mathrm{LR}}$ and $\mathrm{TE}_{\mathrm{LR}}$ did not further contribute to prognostication, we decided to compute the appearance frequency of each qEEG measures within the 10 best combinations (Supplementary Figure S3A). The measure with highest frequency was $\mathrm{MI}_{\mathrm{AP}}$, which appeared in all 10 best combinations. $\mathrm{RDP}_{\mathrm{AP}}, \mathrm{CC}_{\mathrm{LR}}$, and $\mathrm{MI}_{\mathrm{LR}}$ were close second, each appearing in 9/10 of the top combinations. $\mathrm{CC}_{\mathrm{AP}}$ was part of $6 / 10$ best combinations (including the three best), and $\mathrm{TE}_{\mathrm{AP}}$ of $5 / 10$ best combinations. $\mathrm{RDP}_{\mathrm{LR}}$ and $\mathrm{TE}_{\mathrm{LR}}$ had indeed the lowest appearance frequency, 3 and 4 respectively (in particular, these two measures were 
part of none of the four best combinations). All 256 combinations were also tested according to alternative criteria, wherein the false positive rate (FPR) and the false negative rate (FNR) were given different relative weights in defining the performance (Supplementary Figure S3B-G). $\mathrm{RDP}_{\mathrm{AP}}$ was part of the best performing combination in all 6 tested settings, $\mathrm{CC}_{\mathrm{LR}}$ in 5/6 (in the sixth case it was part of the second best combinations), $\mathrm{MI}_{\mathrm{LR}}$ and $\mathrm{MI}_{\mathrm{AP}}$ in $4 / 6, \mathrm{CC}_{\mathrm{AP}}$ and $\mathrm{TE}_{\mathrm{AP}}$ in $3 / 6$, and finally $\mathrm{RDP}_{\mathrm{LR}}$ and $\mathrm{TE}_{\mathrm{LR}}$ in $1 / 6$. In summary, the qEEG measures which were the most frequently included in the 10 best combinations - as measured based on the AUC or based on differently weighted sums of FPR and FNR - were: $\mathrm{RDP}_{\mathrm{AP}}, \mathrm{CC}_{\mathrm{LR}}$, and $\mathrm{MI}_{\mathrm{AP}}$. The least frequently included measures were: $\operatorname{RDP}_{\mathrm{LR}}$ and $\mathrm{TE}_{\mathrm{LR}}$ (Supplementary Figure S3).

The same analysis was performed on the subgroup of patients with hypoxic/anoxic cause of coma. We found two main differences compared to the case where all patients were included. Firstly, the predictive value of the best combination was higher (AUC 0.946; Fig. 5B). Second, the optimal combination contained only 3 different measures, namely $\mathrm{CC}_{\mathrm{LR}}, \mathrm{MI}_{\mathrm{LR}}$, and $\mathrm{TE}_{\mathrm{AP}}$. Here too, other combinations reached a very close score (Supplementary Figure S4A). The measures with highest inclusion frequency in the 10 best combinations were $\mathrm{CC}_{\mathrm{LR}}(9 / 10), \mathrm{TE}_{\mathrm{LR}}(7 / 10)$, and $\mathrm{TE}_{\mathrm{AP}}(6 / 10$, including the 4 best). Also when performances were assessed with various weighted sum of FPR and FNR, we found that the best combinations for prediction of mortality in posthypoxic/postanoxic patients contained on average less measures than the best combinations for all etiologies. One measure was included in the best combination in all settings, namely $\mathrm{MI}_{\mathrm{LR}}$ (Supplementary Figure S4). The measure that had the highest frequency in all settings was $\mathrm{CC}_{\mathrm{LR}}$; the measures the least often included were $\mathrm{RDP}_{\mathrm{LR}}$ and $\mathrm{MI}_{\mathrm{AP}}$ (Supplementary Figure S5).

\section{Discussion}

Because electroencephalography (EEG) directly relates to brain function, this diagnostic tool is of paramount importance in the assessment of patients with impaired consciousness. It is of unquestionable value to recognize a non-convulsive status epilepticus (Oddo et al., 2009; Sutter et al., 2013) and to document response to 
seizure suppressive therapy (Claassen et al., 2013). EEG also contributes to prognosis, as several visually identifiable patterns such as isoelectric voltage, alpha-coma, or burst-suppression with identical bursts have been recognized from early on as being predictor of bad outcome (Bassetti et al., 1996; Cloostermans et al., 2012; Crepeau et al., 2013; Oddo and Rossetti, 2014; Rossetti et al., 2012; Synek, 1990; TjepkemaCloostermans et al., 2015). EEG has been included in several multi-modal diagnostic procedures (Bassetti et al., 1996; Oddo and Rossetti, 2014; Rossetti et al, 2010; Wijdicks et al., 2006). Despite all these advantages, classical EEG has also several limitations, such as the lack of a validated, and generally accepted classification system of patterns, or the dependence on the expertise of the clinician who interprets the EEG (Taccone et al., 2014).

Previous studies demonstrated that qEEG methods - mainly based on spectral decomposition - can circumvent some of these limitations, by recognizing specific patterns of "classical" EEG visual analysis, in the absence of an electroencephalographer, and in a reproducible manner. The goal of the present study was to explore the utility of qEEG for extracting features from the EEG signals that are not easily accessible to a human observer.

We applied four different synchronization measures between EEG signals in the left and right hemisphere (inter-hemispheric axis), and between signals in the anterior and posterior brain regions (intra-hemispheric axis). When considering all patients, regardless of the etiology of coma, we found that five measures showed a statistical difference between patients who were alive at discharge, and patients who deceased while in the ICU: $\mathrm{RDP}_{\mathrm{AP}}, \mathrm{CC}_{\mathrm{LR}}, \mathrm{CC}_{\mathrm{AP}}, \mathrm{TE}_{\mathrm{LR}}$ and $\mathrm{TE}_{\mathrm{AP}}$. In addition, $\mathrm{CC}_{\mathrm{LR}}$ showed a statistical difference between groups of patients with different etiologies of coma. Using a Bayes classifier based on mixture of Gaussians, we found that the combination with best predictive value was composed of $\mathrm{RDP}_{\mathrm{AP}}, \mathrm{CC}_{\mathrm{LR}}, \mathrm{CC}_{\mathrm{AP}}, \mathrm{MI}_{\mathrm{LR}}$, $\mathrm{MI}_{\mathrm{AP}}, \mathrm{TE}_{\mathrm{AP}}$ This combination had a high predictive value as measured with AUC (0.875). When considering only the subgroup of patients with post hypoxic/anoxic encephalopathy, the combination with best predictive value consisted of $\mathrm{CC}_{\mathrm{LR}}, \mathrm{MI}_{\mathrm{LR}}$, and $\mathrm{TE}_{\mathrm{AP}}$. Its predictive value was even higher (AUC 0.946). In both cases (all patients or only hypoxic/anoxic), several other combinations showed an almost equally good prognostic value. 
This study has several limitations. Firstly, the vast majority of non-survivors deceased after withdrawal of life support, leaving open the possibility that some patients might have survived, if they had been given more time. However, we expect this bias to be small, since all patients received the current best medical treatment. Secondly, patients who were alive at discharge were not assessed with respect to long-term functional outcome. Their functional outcome may have been poor, or might have even deceased after a few weeks/months. Assigning these patients to the category good outcome would then be misleading. However, previous studies showed that this bias as well might be relatively small. In a multicentre prospective study on neurological outcome after cardiac arrest and therapeutic hypothermia, 96\% of patients surviving the acute phase were found to have a favourable long-term outcome, defined as a Cerebral Performance Category of 1-2 (Cronberg et al., 2009). In a recently published study on multimodal coma prognostication, the proportion of good outcome in long-time survivors was with $86 \%$ slightly lower (Rossetti and Oddo, 2014); however, the predictive performances for mortality, or for poor neurological recovery, were very similar - both for individual markers and for a multimodal model. Furthermore, our study was retrospective, which has relevant consequences, since not all comatose patients were included, but only those in whom an EEG was requested by the treating physicians in the ICU, probably resulting in higher percentage of epileptic patients, and more severely ill patients. We could not control for medication, which may have influenced our results, even though the sedation with propofol was not significantly different in the group of survivors and non-survivors. Finally, the time at which the EEG was performed also depended exclusively on clinical criteria, and was thus different for each patient. This might be of relevance, since the prognostic value of several EEG patterns depend strongly on the time at which they are recorded (Cloostermans et al., 2012; Sandroni et al., 2014), and future studies will have to investigate the modification of qEEG coupling measures over time.

Currently, qEEG signal coupling measures are not used in clinical practice in the context of coma. Despite the limitations mentioned above, our study shows for the first time that these measures may contribute significantly to the multimodal assessment of comatose patients, especially in the prognostication of post-anoxic 
encephalopathy. Furthermore, the high predictive values obtained in a collective of patients with different coma etiologies, and despite variable delay since coma onset indicate that these methods are particularly robust, and thus appropriate markers for clinical practice.

\subsection{Quantitative vs. visual interpretation}

When analysing an EEG, a trained Electroencephalographer can easily identify single transient signals, evaluate their amplitude, "shape", and spatio-temporal distribution (including synchronicity in case of generalized patterns). He/she will also be able to perform rudimentary spectral analysis. By contrast, humans are very inefficient in performing statistic analysis, a domain in which they are clearly outperformed by computers (Bishop, 2006).

Thus, the two measures that are the most likely to identify similar patterns as a human observer are RDP (which is frequency dependent) and CC (which assesses the similarity of shapes -up to a multiplicative factor). Because it relies on objective criteria, quantitative analysis can nevertheless differ from visual analysis, and is thus likely to provide additional information to mere visual analysis. If we consider for instance the trace in Figure 2a, we clearly identify a left/right asymmetry due to the presence of additional high amplitude waves with frequency of about $1 \mathrm{~Hz}$ on the right hemisphere. However, this did not translate into high value in left-right RDP asymmetry, since most of the power of both hemispheres was in the delta range. The opposite effect is observed in the trace of Figure 2c. At first glance this EEG seems perfectly homogeneous, whereas our analysis shows that it is the EEG trace with the highest left-right RDP asymmetry. This apparent paradox comes from the fact that its spectral decomposition is dominated by peaks at the frequency of the periodic discharges $(2 \mathrm{~Hz})$, and at harmonic frequencies $(4,6$ and $8 \mathrm{~Hz})$. Here, discrete differences in the traces can result in relatively large differences in relative heights of these peaks in the spectrogram (two of which are in the delta band). Cross-correlation also can be difficult to estimate, since elecroencephalographers are more prone to detect "synchrony" (in the classical clinical EEG sense of "occurrence of transients within the same small time window") than mathematically defined correlation (which requires both very precise temporal synchrony and similarity of the wave form). This 
point also can be illustrated in Figure 2c: First, we observe that $\mathrm{CC}_{\mathrm{LR}}$ is extremely high despite a maximal $\mathrm{RDP}_{\mathrm{LR}}$ value. But more interestingly, $\mathrm{CC}_{\mathrm{AP}}$ is low. Here the explanation comes from the anterior-posterior temporal lag of the epileptiform discharges. Since the discharges are of very short duration, the maximal negative and positive values on the frontal and on the parietal derivations did not occur simultaneously, resulting in this low correlation. Note that a phase lag does not automatically mean low correlation. Typical triphasic waves (such as visible in the EEG from Fig 2b) also have an anterior to posterior time lag. But since these waves are of longer duration, the positive deflections still largely coincide, increasing the $\mathrm{CC}_{\mathrm{AP}}$ value.

MI and TE, by contrast, are non linear measures, based on statistical properties of the signal which humans are not able to identify. These measures are the most likely to provide useful additional information to the analysis of EEG compared to visual interpretation. It is thus interesting that both for the case when all patients were analysed, as well as for the subgroup of patients with hypoxic/anoxic etiology of coma, the best combinations contained both linear and non-linear measures. As if the two different classes of methods were providing complementary observations, the combination of which would allow for a more complete EEG analysis. We may thus postulate that combining classical EEG analysis (including for instance reactivity to stimulus) and qEEG (mostly non-linear) coupling measure would further increase the predictive value of EEG. This hypothesis remains to be tested in prospective studies.

\subsection{Pathophysiological interpretations}

Previous studies have shown that synchronization of EEG or fMRI signals is lower during sedation and in comatose patients compared to awake subjects (Boveroux et al., 2010, Cauda et al., 2009; Mäki-Marttunen et al., 2013, Noirhomme et al., 2010). Based on this observation, it seems intuitive that for comatose patients, the lower the correlation, the "deeper" the coma, and the worse the prognosis. However, we have found that this only holds true in the anterior-posterior axis, whereas in the left-right axis a higher correlation is associated with poor outcome. What could be the explanation of this apparent paradox? 
We recall that major causes of coma are: (A) a disruption of cortical activation by the ascending arousal system, (B) extensive and bilateral structural lesions of the brain hemispheres, or (C) a global physiological dysfunction, which can be toxic, metabolic, endocrine, or epileptic (Wijdicks, 2010). These causes are not exclusive; for instance a patient who suffered a cardiac arrest might have cortical and brainstem lesions, while being sedated. Mechanism B might explain our findings in the anteriorposterior axis: It seems plausible that severe white matter or cortical lesions, which are associated with poor outcome, would also contribute to a reduction in intrahemispheric synchronization. Our findings for $\mathrm{TE}_{\mathrm{AP}}$ could be explained by the same mechanism. In awake animals and humans, the frontal-to-parietal information flow has been shown to exceed the parietal-to-frontal flow; this anterior-to posterior flow has also been shown to be selectively attenuated during anaesthesia (Imas et al., 2005; Lee et al., 2009; Lee et al., 2013). We may postulate that a disruption of intrahemispheric connections would result in a further reduction of the anterior-toposterior flow, resulting in an abolished net directionality of information flow, as observed in patients with poor outcome.

Our interpretation of unfavorable prognostic associated with high $\mathrm{CC}_{\mathrm{LR}}$ involves Mechanism A, and postulates a disruption of the ascending activating system. Experimental studies have shown that after cortical deafferentation, the loss of subcortical input to the cortical cells leads to a compensatory increase in short- and long range cortico-cortical connection, and thus to a higher cortical synchronization (Lemieux et al., 2014; Topolnik et al., 2003). By contrast, physiological sleep shows topographical differences, especially between hemispheres (Imbach et al., 2012; Murphy et al., 2011; Roth et al., 1999; Vyazovskiy et al., 2002; Werth et al., 1996;), the extreme case being the dolphins where only one hemisphere sleeps at the time (Mukhametov, 1987). Coma with high interhemispherical synchronization may be associated with poor prognosis, because it differs the most from physiological sleep. To evaluate if our transfer entropy results were consistent with the hypothesis of an increased weight of interhemispheric cortico-cortical connections in patients with unfavorable outcome, we computed the total (from left to right plus from right to left) interhemispheric transfer entropy. We found indeed a higher total information flow in patients who did not survive $(\mathrm{S}=0.122+/-0.02, \mathrm{D}=0.145+/-0.05, \mathrm{p}=0.02)$. By comparison, the difference was not significant in the anterior-posterior axis $(\mathrm{S}=0.113$ 
$+/-0.02, \mathrm{D}=0.117+/-0.06 ; \mathrm{p}=0.38)$. Interestingly, an anisotropy in synchronization has already been described in a study in which both an increase and a decrease in correlation between various brain regions were seen in patients recovering from posttraumatic brain injury (Shields et al., 2007).

In summary, different synchronization levels might reflect different mechanisms of coma. We have shown that these differences could be used in clinical context for diagnostic; they could also be used for future studies on pathophysiology of coma.

\section{Acknowledgement}

This study was supported by the Swiss National Science Foundation (SNF-141055).

The sponsor was not involved in study design, conduction or publication. 


\section{Figure Legends}

Figure 1. Left-right (interhemispheric) and anterio-posterior (intrahemispheric) axis. (A) To assess synchronization between the left and right hemispheres, bivariate quantitative EEG (qEEG) coupling measures are computed between bipolar derivations F3-P3 (left) and F4-P4 (right). (B) Synchronization between the anterior and posterior brain regions are assessed with bivariate qEEG measures between bipolar derivations F3-F4 (anterior) and P3-P4 (posterior).

Figure 2. Four typical examples of coma EEG patterns, with corresponding values of qEEG coupling measures. EEG traces are presented in a longitudinal bipolar montage, which is used in clinical practice (only the paramedian rows are displayed, in blue for the left side, in black for the right side). The value of the 8 different qEEG measures is represented on a normalized scale, a complete bar representing the highest value observed within the 79 subjects $\left(1, \mathrm{RDP}_{\mathrm{LR}} ; 2, \mathrm{RDP}_{\mathrm{AP}} ; 3, \mathrm{CC}_{\mathrm{LR}} ; 4, \mathrm{CC}_{\mathrm{AP}} ; 5, \mathrm{MI}_{\mathrm{LR}} ; 6\right.$, $\mathrm{MI}_{\mathrm{AP}} ; 7, \mathrm{TE}_{\mathrm{LR}} ; 8, \mathrm{TE}_{\mathrm{AP}}$ ). (A) Continuous, diffuse, irregular activity with superimposed delta and subdelta on the right hemisphere in 76 year old male with primarily epileptic (post-ictal) cause of coma (alive and awake at discharge; Patient No. 43 in Supplementary Table S1). (B) Diffuse, irregular theta-alpha activity with isolated triphasic waves, in a 51 year old male, hypoxic cause of coma (alive and awake at discharge; Patient No. 4). (C) Generalized periodic discharges in 59 year old male, hypoxic cause of coma (support withdrawal; Patient No. 2). (D) Burstsuppression with identical bursts in a 28 year old female, hypoxic cause of coma (support withdrawal; Patient No. 25).

Figure 3. Values of coupling measures in patients grouped according to the clinical outcome. Bullets show the mean; bars inside the boxes show the median, top and bottom of boxes show the percentile 25 and 75, and whiskers percentile 5 and 95. (A) $\mathrm{RDP}_{\mathrm{LR}}$ : no differences were observed depending on the outcome. (B) $\mathrm{RDP}_{\mathrm{AP}}$ : the absolute-valued asymmetry in relative delta power was higher in patients who deceased in ICU $(\mathrm{p}=0.00012 *)$. $(\mathbf{C}) \mathrm{CC}_{\mathrm{LR}}$ : a higher interhemispheric correlation was observed in patients who deceased in ICU $(\mathrm{p}=0.0073 *)$. (D) $\mathrm{CC}_{\mathrm{AP}}$ : a higher intrahemispheric correlation was observed in survivor patients $\left(\mathrm{p}=0.026^{*}\right)$. (E) $\mathrm{MI}_{\mathrm{LR}}$ : 
no differences were observed depending on the outcome. (F) $\mathrm{MI}_{\mathrm{AP}}$ : no differences were observed. (G) $\mathrm{TE}_{\mathrm{LR}}$ : he absolute-valued asymmetry in interhemispheric information flow was more pronounced in patients with bad outcome $\left(p=0.04^{*}\right)$. (H) $\mathrm{TE}_{\mathrm{AP}}$ : patients who survived had an information flow more directed from anterior to posterior brain regions $\left(\mathrm{p}=0.015^{*}\right)$. Asterisks $(*)$ indicate statistical significance after control for multiple comparisons.

Figure 4. Values of coupling measures in patients grouped according to the etiology of coma. Bullets show the mean; bars inside the boxes show the median, top and bottom of boxes show the percentile 25 and 75, and whiskers percentile 5 and 95. (A) $\mathrm{RDP}_{\mathrm{LR}}$ : no differences were observed depending on the etiology. (B) $\mathrm{RDP}_{\mathrm{AP}}$ : the difference between hypoxic and epileptic etiology of coma was close to significance level ( $\mathrm{p}=0.053)(\mathbf{C}) \mathrm{CC}_{\mathrm{LR}}$ : the interhemispheric correlation was significantly higher in patients with post hypoxic encephalopathy than in patients with a primarily epileptic or infectious etiology ( $\left.\mathrm{p}=0.00052^{*}\right)$. (D) $\mathrm{CC}_{\mathrm{AP}}$ : the difference in intrahemispheric correlation between patients with hypoxic or epileptic etiology was close to significance ( $\mathrm{p}=0.057)$. $(\mathbf{E}-\mathrm{H})$ For $\mathrm{MI}_{\mathrm{LR}}, \mathrm{MI}_{\mathrm{AP}}, \mathrm{TE}_{\mathrm{LR}}$, and $\mathrm{TE}_{\mathrm{AP}}$ : no differences were observed according to the etiology. Asterisks (*) indicate statistical significance after control for multiple comparisons.

Figure 5. Performance of the classifier. The receiver operating characteristic (ROC) curve of combinations with best predictive value as measured by the area under the ROC curve (AUC) is shown. (A) When considering all patients, the combination with highest AUC was $\mathrm{RDP}_{\mathrm{AP}}, \mathrm{CC}_{\mathrm{LR}}, \mathrm{CC}_{\mathrm{AP}}, \mathrm{MI}_{\mathrm{LR}}, \mathrm{MI}_{\mathrm{AP}}$, and $\mathrm{TE}_{\mathrm{AP}}(\mathrm{AUC}$ 0.875). (B) When considering only patients with post anoxic/hypoxic encephalopathy, the highest AUC was 0.946. It was obtained by the combination $\mathrm{CC}_{\mathrm{LR}}, \mathrm{MI}_{\mathrm{LR}}$, and $\mathrm{TE}_{\mathrm{AP}}$. Black: mean of the 79 leave-one-out trials; grey area: standard deviation. 


\section{References}

Bassetti C, Bomio F, Mathis J, Hess CH. Early prognosis in coma after cardiac arrest: a prospective clinical, electrophysiological and biochemical study of 60 patients. J Neurol Neurosurg Psychiatry 1996;61:610-5.

Bishop CM. Pattern recognition and machine learning. 2006. Springer.

Boveroux P, Vanhaudenhuyse A, Bruno MA, Noirhomme Q, Lauwick S. Breakdown of within- and between-network resting state functional magnetic resonance imaging connectivity during propofol-induced loss of consciousness. Anesthesiology 2010;113:1038-1053.

Cauda F, Micon BM, Sacco K, Duca S, D'Agata F, Geminiani G, et al. Disrupted intrinsic functional connectivity in the vegetative state. J Neurol Neurosurg Psychiatry 2009;80:429-31.

Claassen J, Hirsch LJ, Kreiter KT, Du EY, Connolly ES, Emerson RG, et al. Quantitative continuous EEG for detecting delayed cerebral ischemia in patients with poor-grade subarachnoid hemorrhage. Clin Neurophysiol 2004;115:2699-710.

Claassen J, Taccone FS, Horn P, Holtkamp M, Stocchetti N, Oddo M.

Recommendation on the use of EEG monitoring in critically ill patients. Intensive Care Med 2013;39:1337-51.

Cloostermans MC, de Vos CC, van Putten MJAM. A novel approach for computer assisted EEG monitoring in the adult ICU. Clin Neurophysiol 2011;122:2100-9.

Cloostermans MC, van Meulen FB, Eertman CJ, Hom HW, van Putten MJAM. Continuous electroencephalography monitoring for early prediction of neurological outcome in postanoxic patients after cardiac arrest: a prospective cohort study. Crit Care Med 2012;40:2867-75. 
Cover TM, Thomas JA (eds). Elements of information theory. Hoboken: Wiley Interscience; 2006.

Crepeau AZ, Rabinstein AJ, Fugate JE, Mandrekar J, Wijdicks EF, White RD, et al. Continuous EEG in therapeutic hypothermia after cardiac arrest: prognostic and clinical value. Neurology 2013;80:334-9.

Cronberg T, Lilja G, Rundgren M, Friberg H, Widner H. Long-term neurological outcome after cardiac arrest and therapeutic hypothermia. Resuscitation 2009;80:1119-23.

Daw CS, Finney CEA, Tracy ER. A review of symbolic analysis of experimental data. Rev Sci Instrum 2003;74:915.

Edlow JA, Rabinstein A, Traub SJ, Wijdicks EF. Diagnosis of reversible causes of coma. Lancet 2014;384:2064-76.

Foreman B, Claassen J. Quantitative EEG for the detection of brain ischemia. Crit Care 2012;16:216.

Go AS, Mozaffarian D, Roger VL, Benjamin EJ, Berry JD, Borden WB, et al. American Heart Association Statistics Committee and Stroke Statistics Subcommittee: Heart disease and stroke statistics - 2013 update: a report from the American Heart Association. Circulation 2013;127:e6-e245.

Gotman J, Flanagan D, Zhang J, Rosenblatt B, Bye A, Mizrahi EM. Automatic seizure detection in the newborn: methods and initial evaluation. Electroencephalogr Clin Neurophysiol 1997;103:356-62.

Groppe DM, Urbach TP, Kutas M. Mass univariate analysis of event-related brain potentials/fields I: A critical tutorial review. Psychophysiology 2011. 48:1711-25. 
Hofmeijer J, Tjepkema-Cloostermans MC, van Putten MJAM. Burst-suppression with identical bursts: A distinct EEG pattern with poor outcome in postanoxic coma. Clin Neurophysiol 2014;125:947-54.

Howard RS, Holmes PA, Koutroumanidis MA: Hypoxic-ischemic brain injury. Pract Neurol 2011;11:4-18.

Imas OA, Ropella KM, Ward BD, Wood JD, Hudetz AG. Volatile anesthetics disrupt frontal-posterior recurrent information transfer at gamma frequencies in rat. Neurosci Lett $2005 ; 387: 145-50$.

Imbach LL, Werth E, Kallweit U, Sarnthein J, Scammell TE, Baumann CR. InterHemispheric Oscillations in Human Sleep. PLoS One 2011;7:e48660.

Jordan KG. Emergency EEG and Continuous EEG Monitoring in Acute Ischemic Stroke. J Clin Neurophysiol 2004;21:341-52.

Kamps MJ, Horn J, Oddo M, Fugate JE, Storm C, Cronberg T, et al. Prognostication of neurologic outcome in cardiac arrest patients after mild therapeutic hypothermia: a meta-analysis of the current literature. Intensive Care Med 2013;39:1671-82.

Kaplan PW, Bauer G. Anoxia, Coma, and Brain Dead. In: Schomer DL, Lopes da Silva FH (eds). Niedermeyer's Electroencephalography: basic principles, clinical applications, and related fields. Philadelphia: Wolters Kluwer; 2011. p 435-56.

Kramer MA. A primer on networks in neuroscience. In: Bianchi M, Cash S, Caviness V (eds). Network Approaches to Diseases of the Brain. Danvers: Benthan Publishing; 2010. p 13-20.

Kramer MA, Eden UT, Kolaczyk ED, Zepeda R, Eskandar EN, Cash SS. Coalescence and fragmentation of cortical networks during focal seizures. J Neurosci 2010;30:10076-85. 
King JR, Sitt JDD, Faugeras F, Rohaut B, El Karoui I, Cohen L, et al. Information sharing in the brain indexes consciousness in noncommunicative patients. Curr Biol 2013;23:1914-9.

Lee U, Kim S, Noh GJ, Choi BM, Hwang E, Mashour GA. The directionality and functional organization of frontoparietal connectivity during consciousness and anesthesia in humans. Conscious Cogn 2009;18:1069-78

Lee U, Ku S, Noh G, Baek S, Choi B, Mashour GA. Disruption of frontal-parietal communication by ketamine, propofol, and sevoflurane. Anesthesiology 2013;118:1264-75.

Lemieux M, Chen JY, Lonjers P, Bazhenov M, Timofeev I. The impact of cortical deafferentation on the neocortical slow oscillation. J Neurosci 2014;34:5689-703.

Mäki-Marttunen V, Diez I, Cortes MJ, Chialvo DR, Villarreal MF. Disruption of transfer entropy and inter-hemispheric brain functional connectivity in patients with disorder of consciousness. Front Neuroinform 2013;7:24.

Mukhametov LM. Unihemispheric slow-wave sleep in the Amazonian dolphin, IniaGeoffrensis. Neurosci Lett 1987;79:128-32.

Murphy M, Huber R, Esser S, Riedner BA, Massimini M, Ferrarelli F, et al. The cortical topography of local sleep. Curr Top Med Chem 2011;11:2438-46.

Noirhomme Q, Soddu A, Lehembre R, Vanhaudenhuyse A, Boveroux P. Brain connectivity in pathological and pharmacological coma. Front Syst Neurosci 2010;4:160.

Oddo M, Carrera E, Claassen J, Mayer SA, Hirsch LJ. Continuous electroencephalography in the medical intensive care unit. Crit Care Med 2009;37:2051-6. 
Oddo M, Rossetti AO. Early multimodal outcome prediction after cardiac arrest in patients treated with hypothermia. Crit Care Med 2014;42:1340-7.

Rossetti AO, Oddo M, Logroscino G, Kaplan PW. Prognostication after cardiac arrest and hypothermia: a prospective study. Ann Neurol 2010;67:301-7.

Rossetti AO, Carrera E, Oddo M. Early EEG correlates of neuronal injury after brain anoxia. Neurology 2012;78:796-802.

Roth C, Achermann P, Borbély AA. Frequency and state specific hemispheric asymmetries in the human sleep EEG. Neurosci Lett 1999;271:139-42.

Rulkov NF, Sushchik, MM, Tsimring LS. Generalized synchronization of chaos in directionally coupled chaotic systems. Phys Rev E 1995;51:980-994.

Rummel C, Abela E, Hauf M, Wiest R, Schindler K. Ordinal patterns in epileptic brains: analysis of intracranial EEG and simultaneous EEG-fMRI. Eur Phys J Spec Top 2013;222:569-85.

Rundgren M, Westhall E, Cronberg T, Rosén I, Friberg H. Continuous amplitudeintegrated electroencephalogram predicts outcome in hypothermia-treated cardiac arrest patients. Crit Care Med 2010;38:1838-44.

Sandroni C, Cariou A, Cavallaro F, Cronberg T, Friberg H, Hoedemaekers C, Horn J, Nolan JP, Rossetti AO, Soar J. Prognostication in comatose survivors of cardiac arrest: an advisory statement from the European Resuscitation Council and the European Society of Intensive Care Medicine. Intensive Care Med 2014;40:1816-31.

Schindler K, Leung H, Elger CE, Lehnertz K. Assessing seizure dynamics by analysing the correlation structure of multichannel intracranial EEG. Brain 2007;130:65-77.

Schreiber T. Measuring information transfer. Phys Rev Lett 2000;85:461-464. 
Shields DC, Leiphart JW, McArthur DL, Vespa PM, LeVan Quyen M, Martinerie J, et al. Cortical synchrony changes detected by scalp electrode electroencephalograph as traumatic brain injury patients emerge from coma. Surg Neurol 2007;67:354-9.

Sitt JD, King JR, El Karoui I, Rohaut B, Faugeras F, Gramfort A, et al. Large scale screening of neural signatures of consciousness in patients in a vegetative or minimally conscious state. Brain 2014;137:2258-70.

Stam CJ, van Dijk BW. Synchronization likelihood: an unbiased measure of generalized synchronization in multivariate data sets. Physica D 2002;163:236-251.

Stam CJ, van Straaten EC. The organization of physiological brain networks. Clin Neurophysiol 2012;123:1067-87.

Staniek M, Lehnertz K. Symbolic transfer entropy. Phys Rev Lett 2008;100:158101.

Sutter R, Stevens RD, Kaplan PW. Continuous electroencephalographic monitoring in critically ill patients: indications, limitations, and strategies. Crit Care Med 2013;41:1124-32.

Synek VM. Value of a revised EEG coma scale for prognosis after cerebral anoxia and diffuse head injury. Clin Electroencephalogr 1990;21:25-30.

Taccone F, Cronberg T, Friberg H, Greer D, Horn J, Oddo M, et al. How to assess prognosis after cardiac arrest and therapeutic hypothermia. Crit Care 2014;18:202.

Tjepkema-Cloostermans MC, van Meulen FB, Meinsma G, van Putten MJAM. Cerebral Recovery Index (CRI) for early prognosis in patients after cardiac arrest. Crit Care 2013;17:R252.

Tjepkema-Cloostermans MC, Hofmeijer J, Trof RJ, Blans MJ, Beishuizen A, van Putten MJAM. Electroencephalogram predicts outcome in patients with postanoxic coma during mild therapeutic hypothermia. Crit Care Med 2014;43:159-167. 
Topolnik L, Steriade M, Timofeev I. Partial cortical deafferentation promotes development of paroxysmal activity. Cereb Cortex 2003;13:883-93.

van Diessen E, Diederen SJH, Braun KPJ, Jansen FE, Stam CJ. Functional and structural brain networks in epilepsy: what have we learned? Epilepsia 2013;54:185565.

van Putten, MJAM, Tavy DLJ. Continuous quantitative EEG monitoring in hemispheric stroke patients using the brain symmetry index. Stroke 2004;35:2489-92.

van Putten MJAM, Kind T, Visser F, Lagerburg V. Detecting temporal lobe seizures from scalp EEG recordings: a comparison of various features. Clin Neurophysiol 2005;116:2480-9.

van Putten, MJAM. The revised brain symmetry index. Clin Neurophysiol 2007;118:2362-7.

Vyazovskiy VV, Olcese U, Hanlon EC, Nir Y, Cirelli C, Tononi G. Local sleep in awake rats. Nature 2011;472:443-7.

Werth E, Achermann P, Borbély AA. Brain topography of the human sleep EEG: antero-posterior shifts of spectral power. Neuroreport 1996;8:123-7.

Wijdicks EFM. Coma. J Neurol Neurosurg Psychiatry 2010;10:51-60.

Wijdicks EF, Hijdra A, Young GB, Bassetti CL, Wiebe S. Practice parameter: prediction of outcome in comatose survivors after cardiopulmonary resuscitation (an evidence-based review): report of the Quality Standards Subcommittee of the American Academy of Neurology. Neurology 2006;67:203-10.

Young GB. Neurologic prognosis after cardiac arrest. N Engl J Med 2009;361:60511. 
Zubler F, Gast H, Abela E, Rummel C, Hauf M, Wiest R, et al. Detecting functional hubs of ictogenic networks. Brain Topogr 2014;28:305-17 

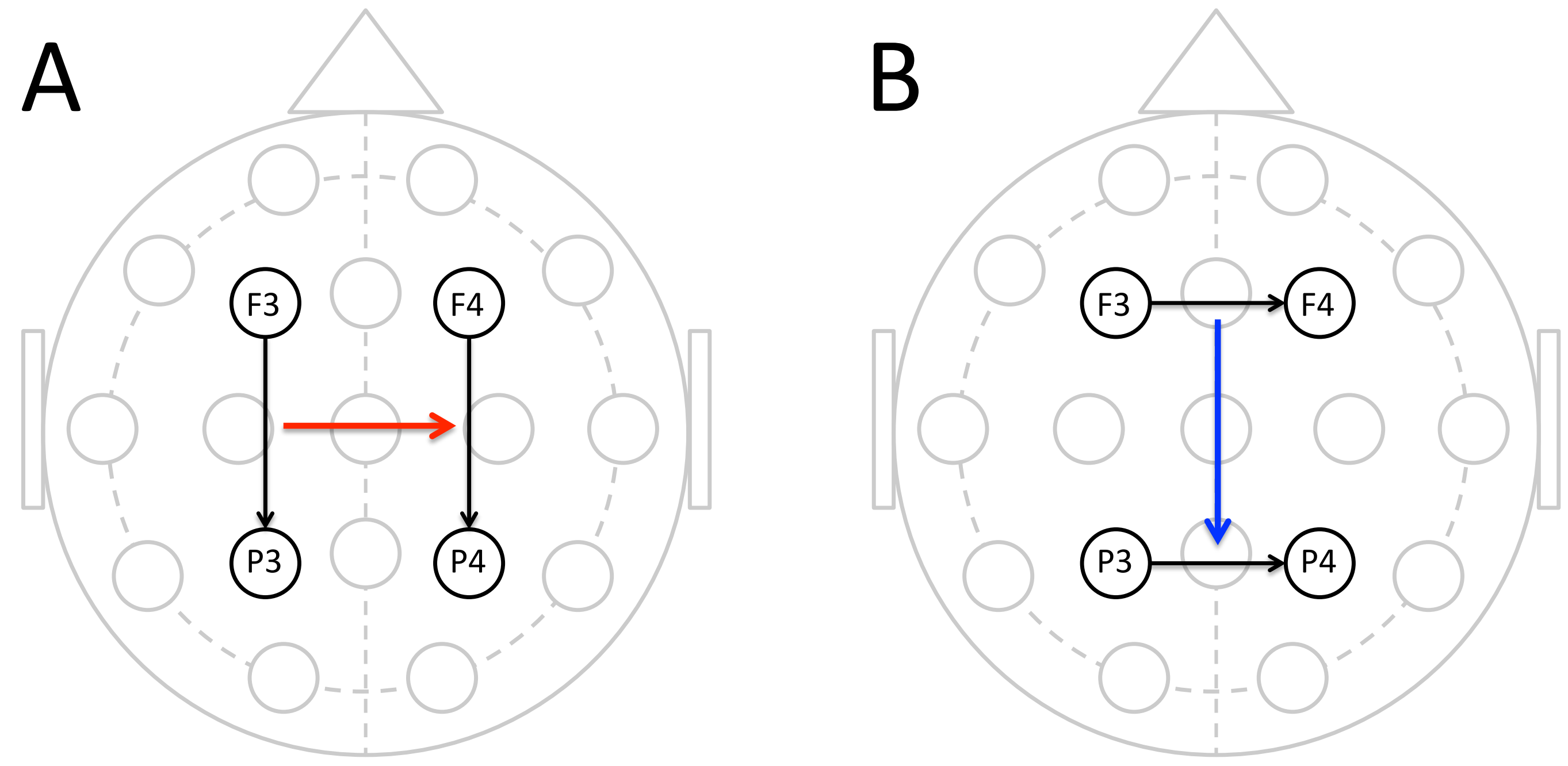


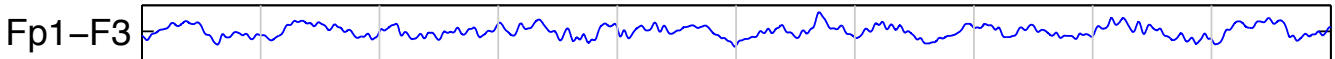
F3-C3 3 = 3 C3-P3 = =

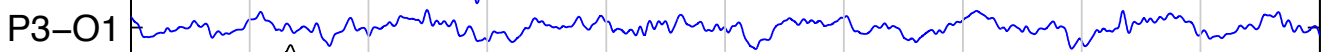

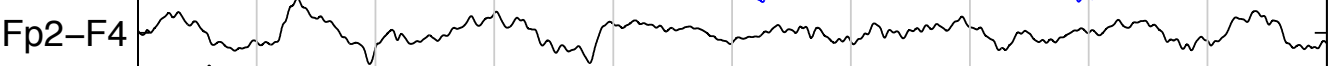
F4-C4 4

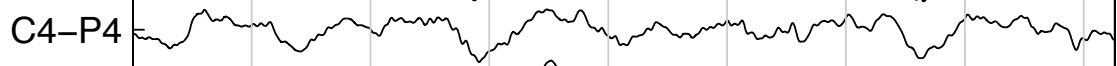

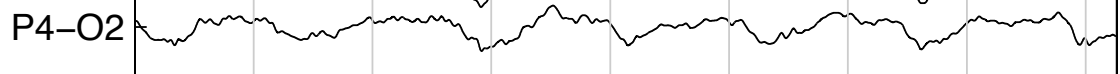

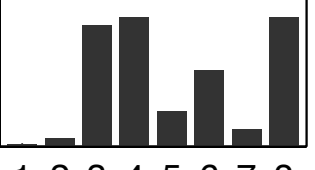

12345678

B

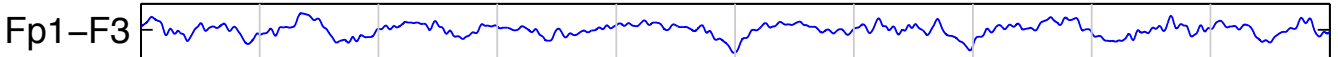

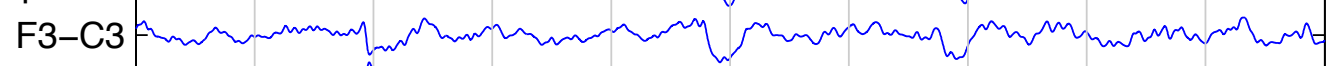

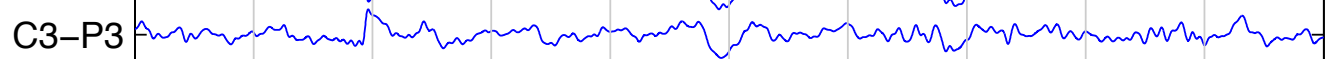

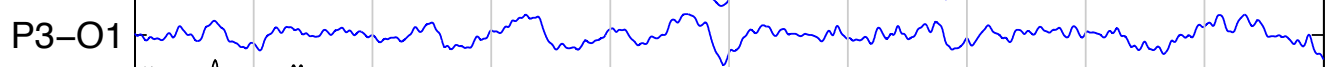

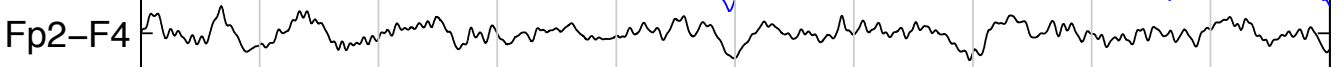
F4-C4 amom

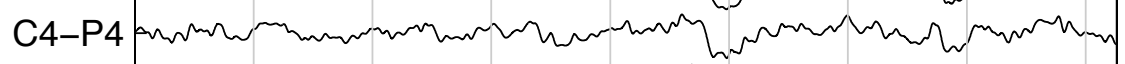

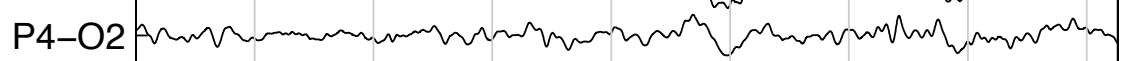

C

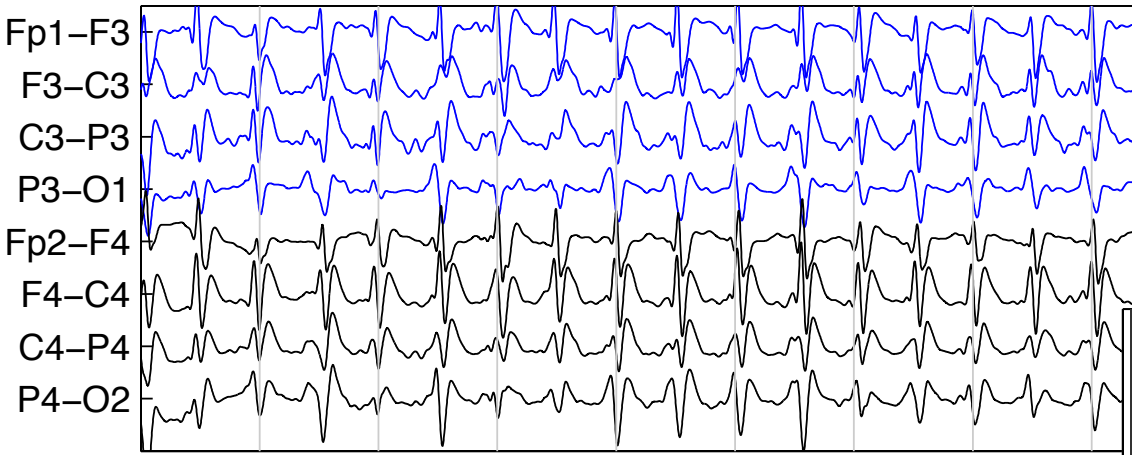

D

12345678

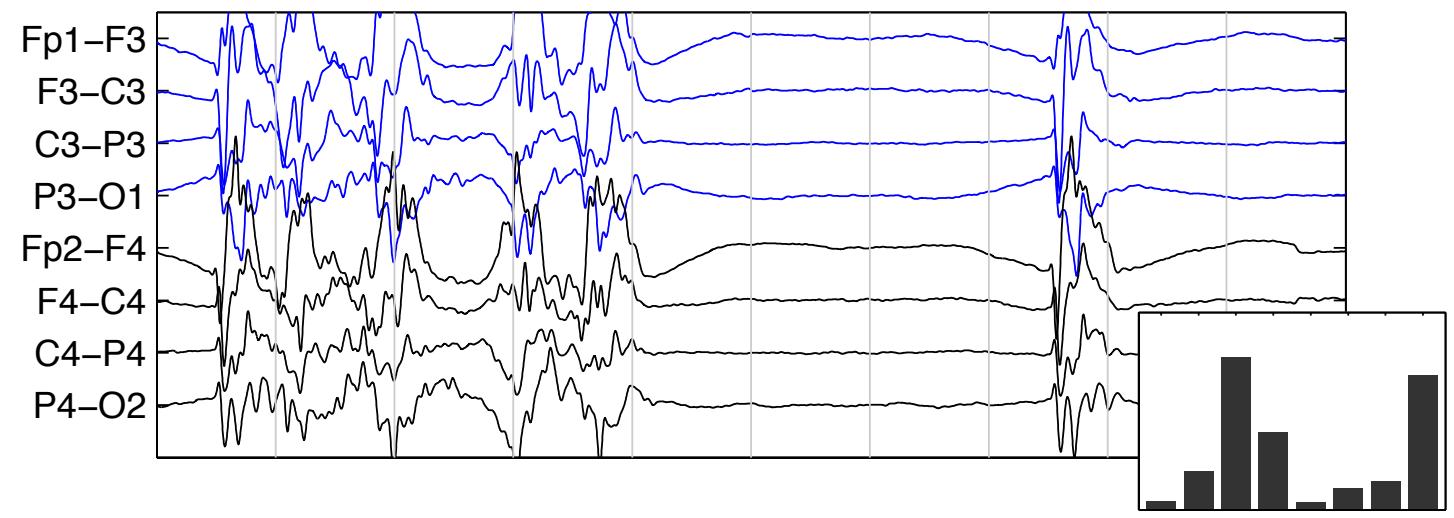

12345678 


\section{O Survivors \\ O Deceased in ICU}

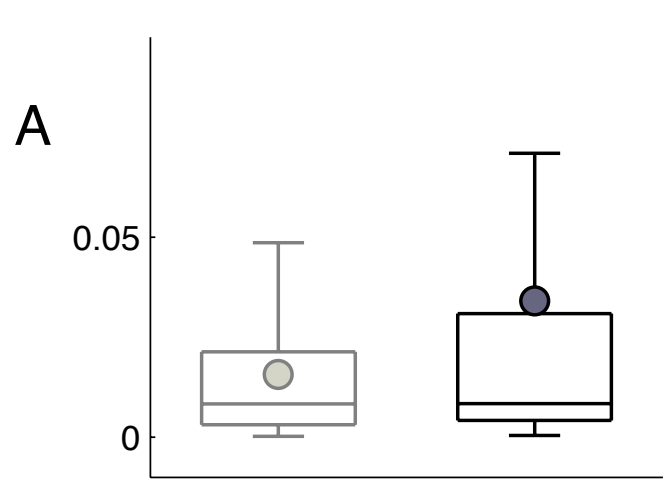

Relative delta power, $\mathrm{L}-\mathrm{R}$

*

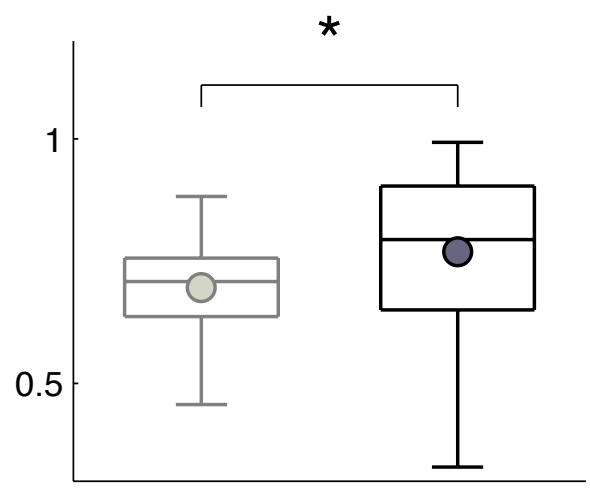

Cross-correlation, L-R

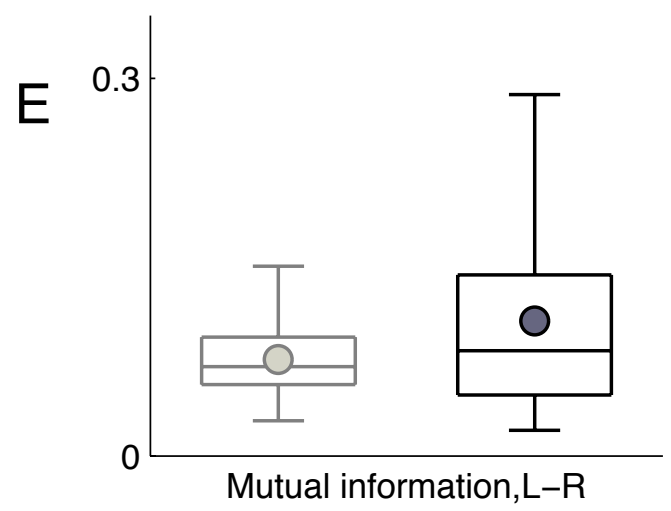

G

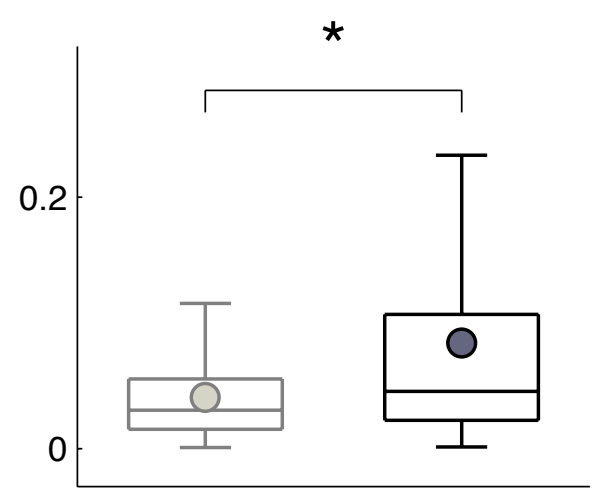

Transfer entropy,L-R

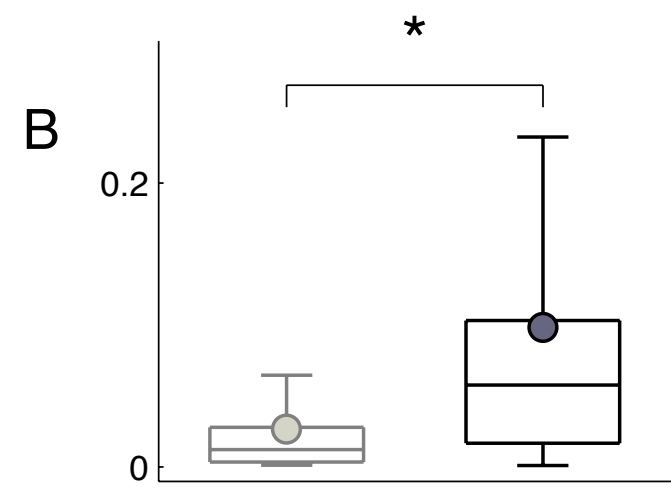

Relative delta power, A-P

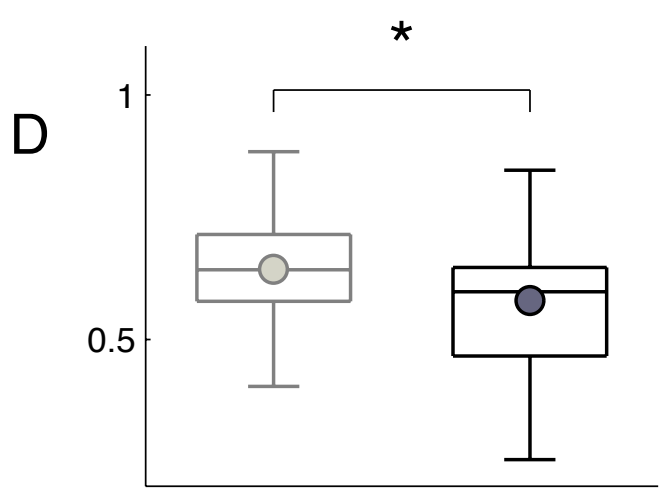

Cross-correlation, A-P
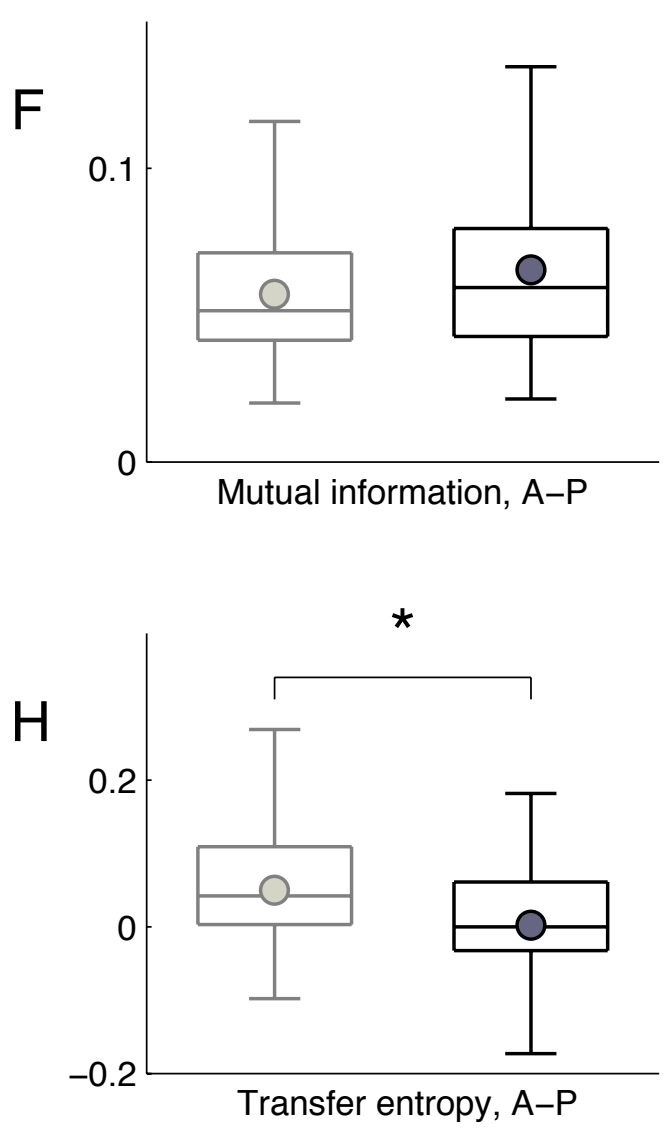


\section{O Hypoxia/Anoxia \\ $\diamond$ Epilepsy \\ $\nabla$ Infection \\ 枝 Metabolic/Medicamentous}

A
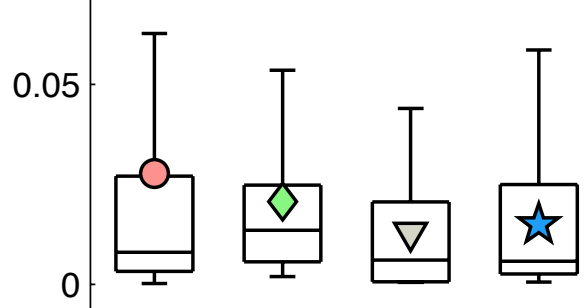

Relative delta power, L-R

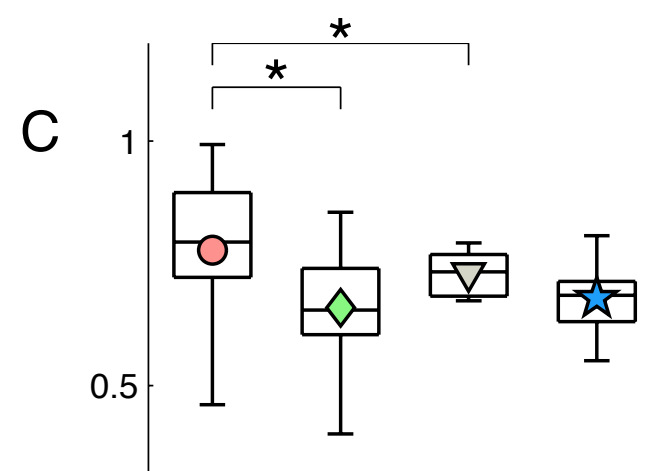

Cross-correlation, L-R
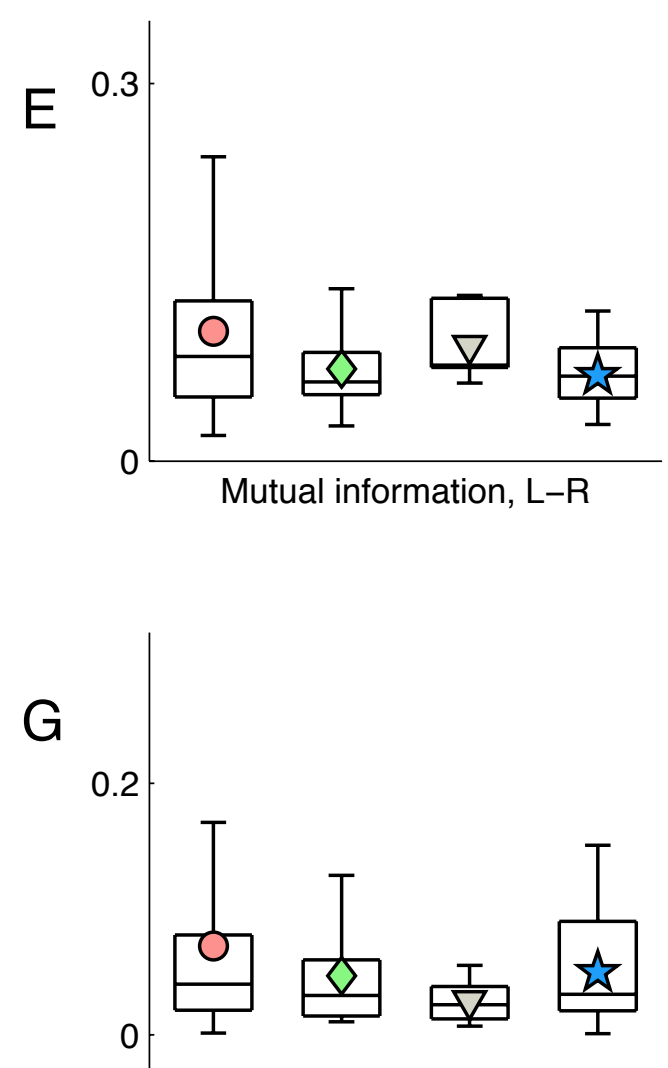

Transfer entropy, L-R

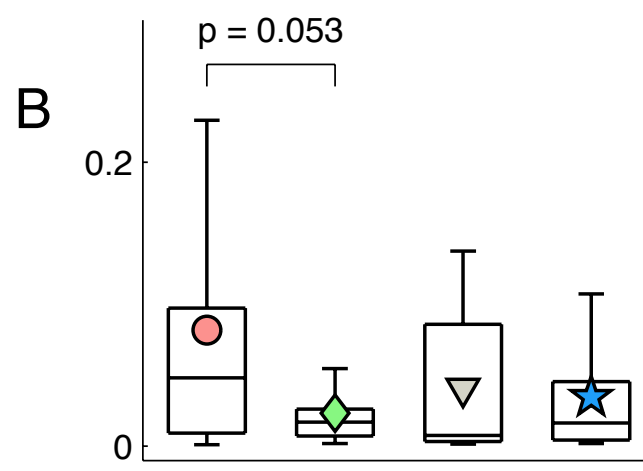

Relative delta power, A-P

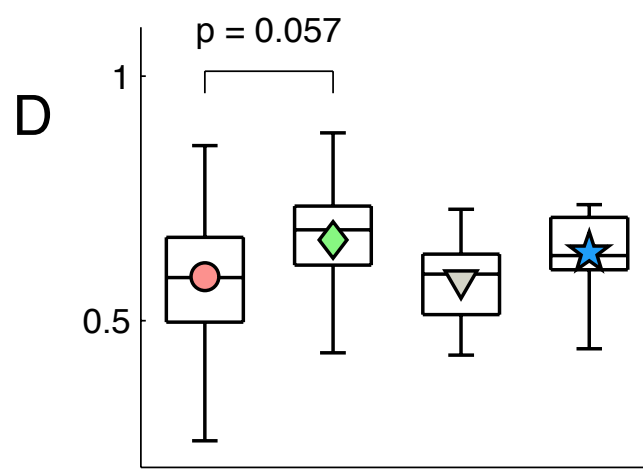

Cross-correlation, A-P
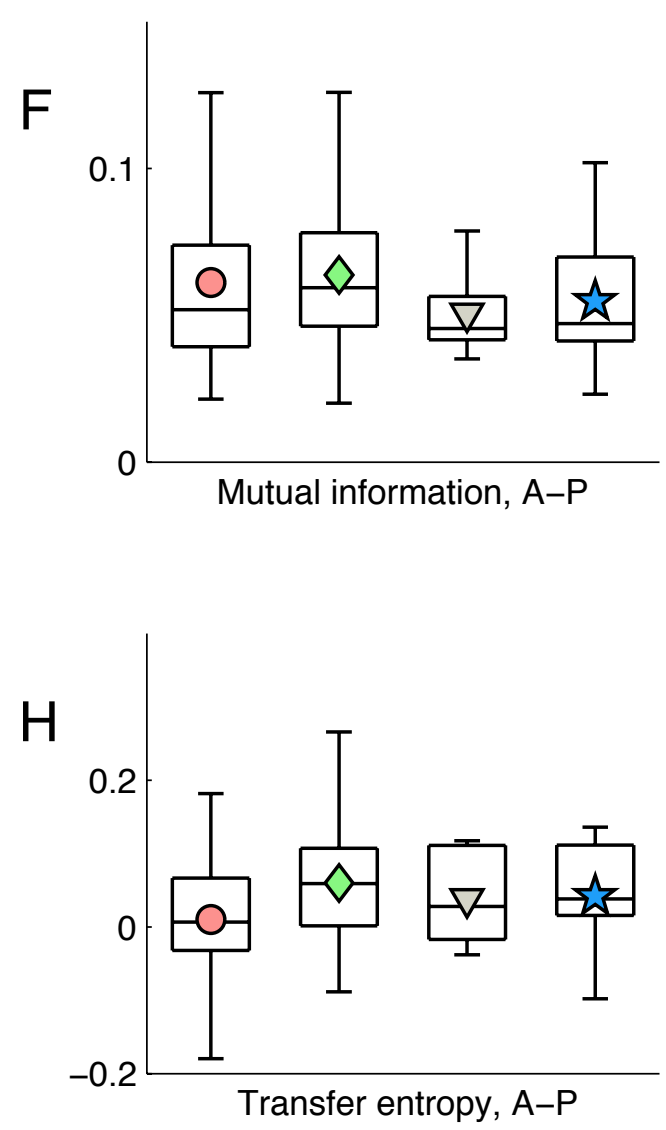


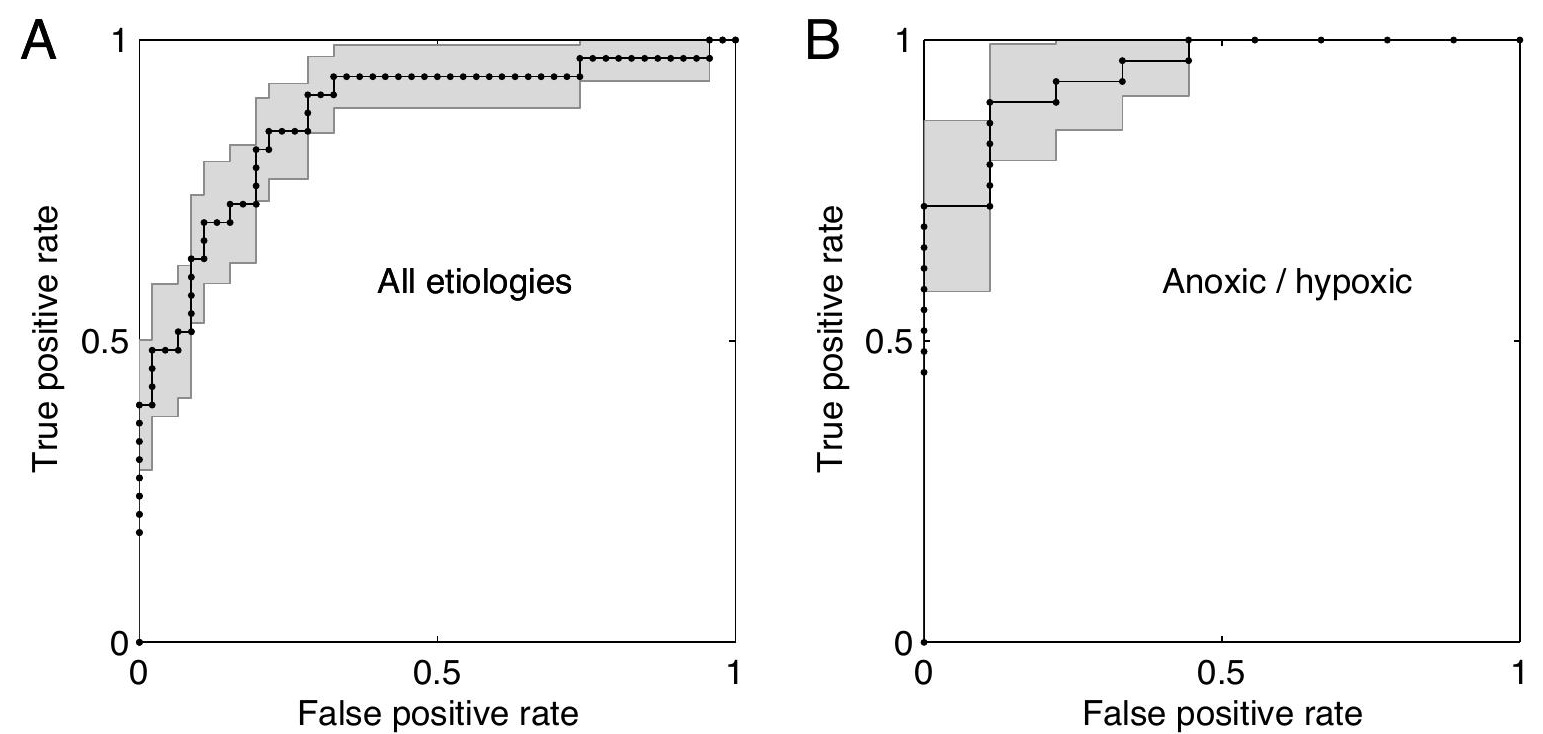

